\title{
A guided Internet-delivered intervention for adjustment disorders: A randomized controlled trial
}

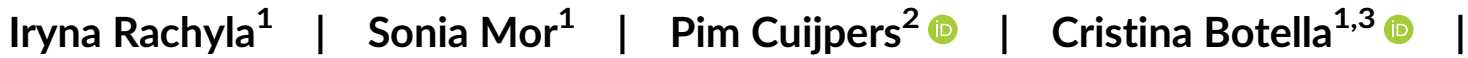 \\ Diana Castilla $^{3,4}$ @ | Soledad Quero ${ }^{1,3}$ ( \\ ${ }^{1}$ Department of Basic, Clinical Psychology and Psychobiology, Universitat Jaume I, Castellón, Spain \\ ${ }^{2}$ Department of Clinical, Neuro and Developmental Psychology, Amsterdam Public Health research institute, Vrije Universiteit Amsterdam, Amsterdam, \\ The Netherlands \\ ${ }^{3}$ CIBER of Physiopathology of Obesity and Nutrition (CIBERObn), Madrid, Spain \\ ${ }^{4}$ Department of Personality, Evaluation and Psychological Treatment, Universidad de Valencia, Valencia, Spain
}

\section{Correspondence}

Soledad Quero, Department of Basic, Clinical Psychology and Psychobiology, Universitat Jaume I, Castellón, Spain.

Email: squero@uji.es

Funding information

Instituto de Salud Carlos III, Grant/Award Number: ISC III CB06 03/0052; Generalitat Valenciana, Grant/Award Number: VALi+d: ACIF/2015/181; Universitat Jaume I, Grant/Award Number: UJI-2018-57;

Ministerio de Economía y Competitividad, Grant/Award Number: PSI2013-41783-R
Evidence of self-help interventions for adjustment disorder (AjD) is limited. This study aims at testing in a randomized controlled trial (RCT) the effectiveness of a disorderspecific, Internet-delivered cognitive-behavioural therapy (ICBT) intervention for AjD. Participants were randomly allocated to either an ICBT with brief weekly telephone support $(n=34)$ or a waiting list group $(n=34)$. Beck's inventories for depression and anxiety were used as primary outcomes. The secondary outcomes were AjD symptoms, post-traumatic growth, positive and negative affect, and quality of life. In all, $76.5 \%$ of the participants completed the intervention. Compared with the control group, participants in the intervention condition showed significantly greater improvement in all outcomes (Cohen's $d$ ranged from 0.54 to 1.21) except in anxiety symptoms measured by Beck Anxiety Inventory $(d=0.27)$. Only ICBT group showed a significant improvement in post-traumatic growth, positive and negative affect, and quality of life. The number of cases that achieved clinically meaningful change in all outcome measures was also higher in the ICBT group. All therapeutic gains were maintained at 3-, 6- and 12-month follow-ups. The current study provides evidence on the effectiveness of ICBT interventions to reduce the impact of AjD. Results suggest that brief self-help intervention with minimal therapist support is more effective than the mere passage of time in reducing the distress symptoms associated to the disorder and also can confer additional benefits.

\section{KEYWORDS}

adjustment disorder, CBT, effectiveness, Internet-delivered interventions, randomized controlled trial

\section{INTRODUCTION}

Adjustment disorder (AjD) is one of the most common psychiatric disorders (Evans et al., 2013). It is characterized by clinically significant emotional and behavioural symptoms in response to an identifiable stressful event (American Psychiatric Association, 2013). Constant worry or rumination about the stressor and/or its implications and a failure to adapt are two core symptoms of AjD (World Health Organization [WHO], 2018). It is considered a mild condition situated between normalcy and pathology (Fernández et al., 2012; O'Donnell 
et al., 2016). However, although the clinical symptoms of AjD are milder than in other anxiety and affective disorders, they are severe enough to cause important distress, functional impairment, low quality of life and even suicidal ideation and behaviour (Casey, Jabbar, O'Leary, \& Doherty, 2015; Fegan \& Doherty, 2019).

Previous research suggests that self-help interventions might be particularly useful for the treatment of AjD, given its often transient nature (Maercker, Bachem, Lorenz, Moser, \& Berger, 2015). Thus, low-threshold interventions are recommended to prevent the chronicity and worsening of symptoms or the development of a more severe disorder (Bachem \& Casey, 2018). Self-help interventions, especially those delivered over the Internet, are already being used effectively for the treatment of other mental disorders, such as depression or anxiety disorders (Andrews et al., 2018), producing similar effects to those of face-to-face therapy (Carlbring, Andersson, Cuijpers, Riperd, $\&$ Hedman-Lagerlöfh, 2018). Their use offers important advantages in terms of reach, flexibility, cost saving and confidentiality (Griffiths, Lindenmeyer, Powell, Lowe, \& Thorogood, 2006; Musiat \& Tarrier, 2014). Different amounts of therapist contact may be included. Guided interventions generally show better outcomes and lower dropout rates than completely unguided programs (Cuijpers, Noma, Karyotaki, Cipriani, \& Furukawa, 2019).

To date, four randomized controlled trials (RCTs) have been conducted to test the effectiveness of self-help interventions for the treatment of AjD (Bachem \& Maercker, 2016; Eimontas, Rimsaite, Gegieckaite, Zelviene, \& Kazlauskas, 2017; Lindsäter et al., 2018; Moser, Bachem, Berger, \& Maercker, 2019). The interventions tested in all of these trials were brief, ranging from four to six modules, except the one conducted by Lindsäter et al. (2018), which included 12 modules. All of them showed clinical improvement in patients who received the intended treatment. Two of the trials, which included completely unguided interventions, reported dropout rates of more than 65\% (Eimontas et al., 2017; Moser et al., 2019), thus limiting the generalizability of the findings. Lower dropout rates were found when the intervention was focused on a specific subgroup of patients (burglary victims) (Bachem \& Maercker, 2016) and when therapist support was provided (Lindsäter et al., 2018). However, only one of the four studies used an assessment interview for the clinical diagnosis of AjD (Lindsäter et al., 2018), whereas the other three used self-report questionnaires to assess the level of AjD symptoms. Therefore, more research is needed in order to draw more solid conclusions.

Given the potential clinical usefulness of Internet-delivered interventions for the treatment of mental disorders in general and AjD in particular, the TAO (Spanish acronym for 'Adjustment Disorders Online') program was developed. To our knowledge, TAO is the first Internet-delivered, disorder-specific intervention for AjD in Spanish. It is based on a manualized intervention protocol for AjD (Botella, Baños, \& Guillén, 2008) that has shown efficacy in several previous studies (Baños et al., 2011; Quero et al., 2017; Quero et al., 2019; Quero et al., 2019). Before its adaptation to an online format, the protocol was reviewed and optimized by including additional treatment components (Rachyla et al., 2018). TAO is a brief program composed

\section{Key Practitioner Message}

- Internet-based CBT can reduce the negative impact of adjustment disorder, at short and long term.

- Internet-based CBT can be an effective way to provide psychological treatment to those who need it.

- Such interventions may contribute considerably to overcoming existing barriers to high-quality mental health services, reduce the burden associated with these disorders and may prevent relapse or worsening of symptoms.

of six modules, and it has the advantage of being suitable for people affected by all kinds of stressors. The aim of the present work is to report the results of the RCT conducted to compare the efficacy of TAO (combined with brief telephone support) to a waiting list $(\mathrm{WL})$ control group in a clinical sample with AjD.

\section{2 | METHODS}

\subsection{Study design}

The study was a two-armed RCT comparing an intervention group that received an ICBT intervention with weekly telephone support with a 7-week WL control group. The allocation was performed by an independent researcher who was not involved in the study, using Epidat software (Version 4.1). Block randomization was used to maintain a balance between the trial groups. The power analysis conducted with G*Power 3 software (Faul, Erdfelder, Buchner, \& Lang, 2007) before the study began revealed that a sample of 52 participants ( 26 per group) was needed to detect an effect size of 0.70 with a power of 0.80 and a one-tailed alpha of 0.05 . Because no similar studies had been published before the start of the trial, the effect size was determined in line with those found in previous studies exploring the efficacy of ICBT interventions in patients with similar mood, anxiety and stress-related symptoms (Berger, Boettcher, \& Caspar, 2014; Berger, Hämmerli, Gubser, Andersson, \& Caspar, 2011; Ivarsson et al., 2014). In accordance with literature review on adherence to ICBT (Van Ballegooijen et al., 2014), a dropout rate of $30 \%$, was anticipated. Thereby, a sample of 68 participants (34 per group) was recruited. Assessments were conducted at baseline, postintervention and three follow-up moments (3, 6 and 12 months).

This trial was registered at the ClinicalTrial.gov database (NCT02758418, May 2, 2016). Further details about the development of TAO and the RCT can be found in the study protocol published elsewhere (Rachyla et al., 2018).

The authors assert that all the procedures contributing to this work comply with the ethical standards of the relevant national and institutional committees on human experimentation and the 1975 
Declaration of Helsinki, as revised in 2008. The protocol for the current RCT was approved by the Ethical Committee of Universitat Jaume I.

\section{2 | Participants, recruitment and eligibility criteria}

Participants were recruited through advertisements in the local media (radio and newspaper), on social networks (Facebook, Instagram, Google+ and online health forums) and on campus noticeboards and websites. An informative e-mail was sent to all the members of the university community at Universitat Jaume I and Universitat de València. Potential participants were also derived from the Emotional Disorder Clinic of Universitat Jaume I and other collaborating centres (Spanish Red Cross and Spanish Association Against Cancer).

People interested in participating sent an e-mail to the indicated address or called the number provided. All requests were answered within $24 \mathrm{~h}$. A telephone interview was arranged to explain the terms of the clinical trial and check the fulfilment of the eligibility criteria. Inclusion criteria were as follows: (i) age $\geq 18$ years; (ii) meeting DSM-5 criteria for AjD; (iii) ability to understand and read Spanish; (iv) ability to use a computer and having access to the Internet; and (v) having an e-mail address. The following criteria led to exclusion: (i) presence of risk of suicide or self-destructive behaviours; (ii) receiving psychological treatment for $\mathrm{AjD}$; (iii) presence of another severe mental disorder (substance abuse or dependence, psychotic disorder, dementia or bipolar disorder); (iv) presence of a severe personality disorder or illness; and (v) an increase and/or change in the medication during the study period.

All participants underwent a semistructured diagnostic interview for AjDs conducted by a psychologist with a master's degree. The interview was designed by the group to detect the presence of stressful events and their impact on patients' lives and well-being and to rule out any other problems such as depression or generalized anxiety disorder. People who met the eligibility criteria had to sign an online informed consent form before randomization.

\section{$2.3 \mid$ Measures}

During the RCT, no face-to-face contact was established with participants. All outcome measures were completed online.

\subsection{1 | Primary outcomes}

In the absence of validated instruments for the diagnosis and assessment of $\mathrm{AjD}$ at the beginning of the trial, the Beck Depression Inventory-Second Edition (BDI-II) (Beck, Steer, \& Brown, 1996) and the Beck Anxiety Inventory (BAI) (Beck \& Steer, 1990) were used as primary outcome measures for the assessment of depression and anxiety symptoms, respectively. Literature suggested that these symptoms represented common manifestations of AjD (Maercker et al., 2013b). Both instruments, $\mathrm{BDI}$ and $\mathrm{BAI}$, are widely used in research as well as in clinical settings, mostly due to their strong psychometric properties (Piotrowski, 1999; Richter, Werner, Heerlein, Kraus, \& Sauer, 1998). Internal consistency in the current sample was good for both measures $(0.87$ for $\mathrm{BDI}$ and 0.88 for $\mathrm{BAl})$.

\subsection{2 | Secondary outcomes}

The Inventory of Stress and Loss (ISL) (Quero et al., 2019) was used to assess the degree to which the stressor (or its consequences) interfered in the respondent's life. This 17-item self-report inventory was developed by the research team in response to the lack of instruments to assess AjD symptoms. The items, rated on a 5-point scale $(0=$ never; 1 = rarely; 2 = sometimes; 3 = often; and 4 = always), were adapted from the Complicated Grief Inventory (Prigerson et al., 1995) and include statements such as 'I think about this person/situation so much that it's hard for me to do things I normally do'. However, because only preliminary validation was performed prior to the commencement of the clinical trial, it was decided not to include this instrument as a primary outcome measure. Cronbach's $\alpha$ of 0.90 was obtained in the current sample. Additionally, the Posttraumatic Growth Inventory (PTGI) (Tadeschi \& Calhoun, 1996), which explores possible positive change experienced as a consequence of the stressful event, the Positive and Negative Affect Scale (PANAS) (Watson, Clark, \& Tellegen, 1988), which assesses the presence of positive and negative emotions, and the Multidimensional Quality of Life Questionnaire (MQLI) (Mezzich et al., 2000), which assesses quality of life, were administered to evaluate the possible additional effects of the intervention. Again, good internal consistency reliability indexes were obtained in the current sample for all these measures (0.91 for PTGI; 0.93 for PANAS Positive; 0.86 for PANAS Negative; and 0.82 for MQLI). A more detailed description of these instruments can be found in the study protocol (Rachyla et al., 2018).

\section{$2.4 \quad$ Treatment and therapists}

Patients received an ICBT intervention based on a structured treatment protocol that combines cognitive-behavioural therapy (CBT), positive psychology techniques and mindful awareness. The treatment protocol was called TAO and consisted of seven sequential modules: 'Starting the program', 'Understanding emotional reactions', 'Learning to deal with negative emotions', 'Accepting problems', 'Learning from problems', 'Changing the meaning of problems' and 'Relapse prevention'.

The key objective of TAO is the emotional elaboration and processing of the stressful event, which is achieved through acceptance, confrontation and developing a new meaning for the problematic situation. The Book of Life is a therapeutic activity focused 
on this objective. It is a personal diary devoted to the stressful event and its impact on the life of the person who experienced it. The Book of Life is a writing exercise, but it also includes the use of symbols, metaphors, pictures and music. Apart from the elaboration component, TAO also includes motivation for change, psychoeducation about common reactions to the stressful event and the positive contribution of problems, behaviour activation, a slow breathing technique, exposure, problem-solving, mindfulness, development of personal strengths and optimism towards the future, and relapse prevention.

The content was presented through texts, videos, pictures and vignettes (see Figure 1). In order to facilitate the implementation of the learned skills, several practical activities were suggested, accompanied by illustrative examples and downloadable worksheets.

The treatment lasted between 7 and 10 weeks. Because guided ICBT interventions usually lead to better outcomes and lower attrition rates (Andersson \& Titov, 2014; Cuijpers et al., 2019), participants received weekly telephone support during the intervention period. The support consisted of a short telephone call (maximum $10 \mathrm{~min}$ ) aimed at clarifying doubts, reinforcing the work accomplished and encouraging patients to keep working. No additional clinical content or counselling was provided.

Only two therapists participated in the study. Both of them were PhD students with a master's degree and at least 1 year of clinical experience.

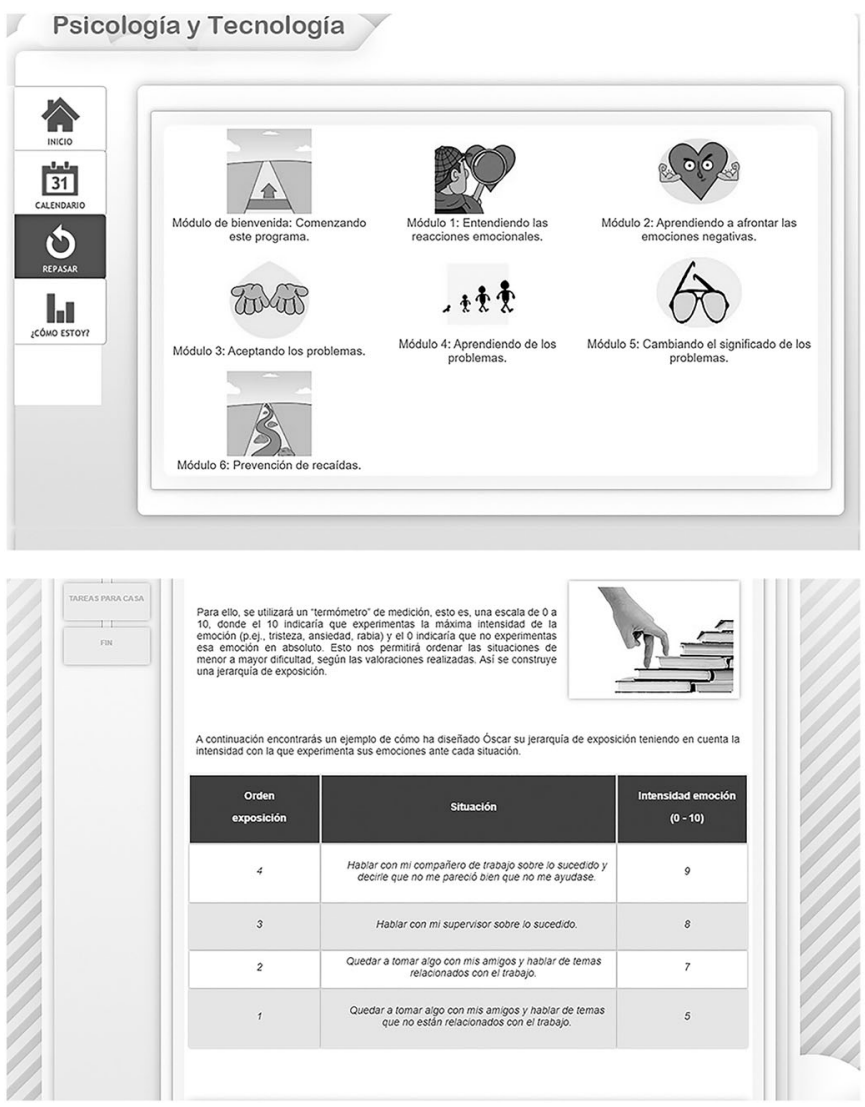

\subsection{Statistical analyses}

Baseline differences between the study groups were examined using chi-squared tests and independent sample $t$ tests for categorical and continuous data, respectively. Repeated-measures analyses of variance (ANOVAs) were conducted to explore changes in the outcome measures from baseline to postintervention in both study groups (using assessment moment as within-group factor and experimental condition as between-group factor) and explore the stability of treatment gains over time in the intervention group. Repeated-measures analyses were performed using linear mixed-effects models (Salim, Mackinnon, Christensen, \& Griffiths, 2008). This approach was chosen because it allows intention-to-treat analyses using the full dataset, thus reducing the biases and loss of power caused by the simple deletion or random imputation of incomplete data. The significance levels were corrected using Bonferroni adjustment in order to reduce type 1 error. Little's test was conducted to verify the random distribution of missing cases. Within- and between-group effect sizes were estimated by computing Cohen's $d$ indices (Cohen, 1988). The clinical significance of the change observed in the scores on the outcome measures from one assessment moment (baseline) to another (post-treatment and follow-up) was determined by calculating the Reliable Change Indexes (Jacobson \& Truax, 1991).

All statistical analyses were conducted using SPSS Statistics Version 22.0 for Windows and following SPIRIT (Chan, Tetzlaff,

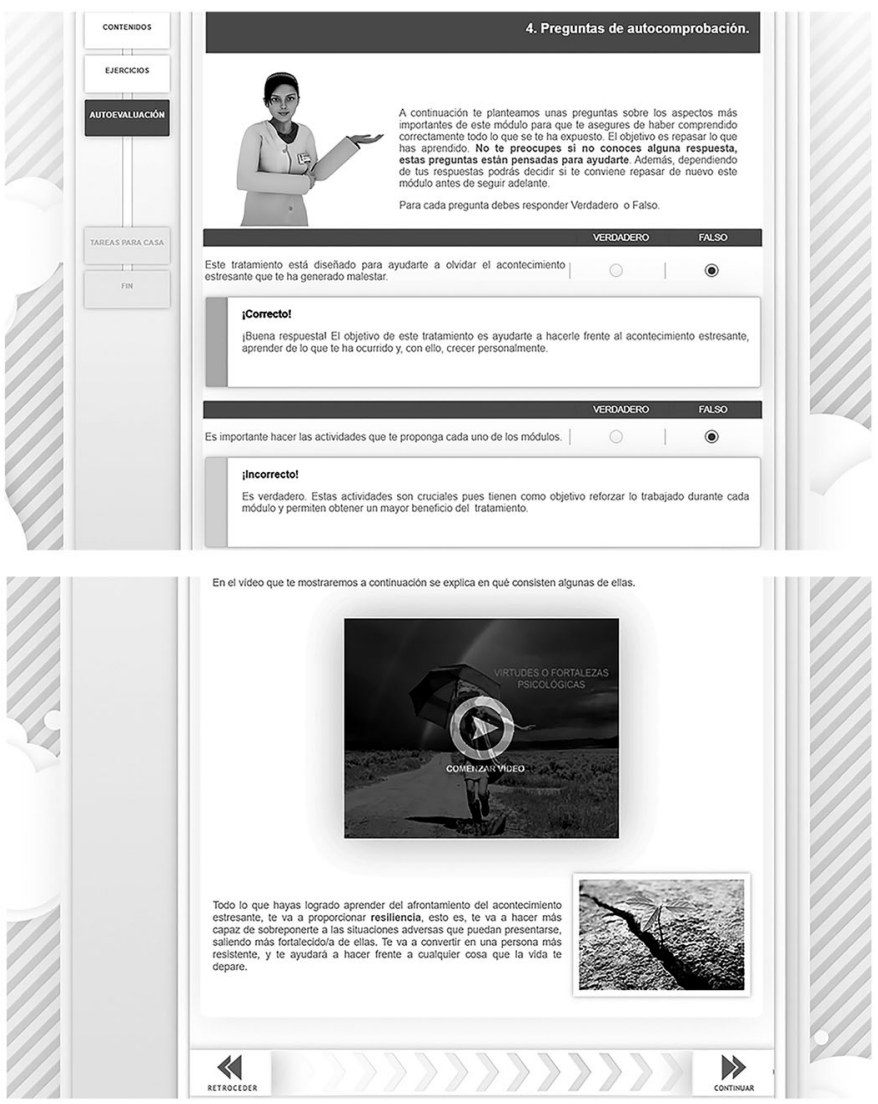

FIGURE 1 TAO screenshots 
Altman, et al., 2013; Chan, Tetzlaff, Gøtzsche, et al., 2013) and CONSORT (Eysenbach, 2011; Moher et al., 2010) guideline recommendations.

\section{3 | RESULTS}

\section{1 | Participant flow and attrition}

Recruitment was carried out between May 2015 and March 2018. Figure 2 shows the flow of participants through each stage of the trial. As the figure shows, 26 out of 34 participants in the ICBT group (76.5\%) completed all seven TAO modules. In the control group, 29 out of 34 completed the baseline assessment, and 26 completed the post-WL assessment. Because the postintervention assessment was performed only when the last TAO module had been completed, participants who had not finished the whole program were considered dropouts. Of the eight participants who were considered dropouts, one $(2.9 \%)$ completed only the first welcome module, three $(8.8 \%)$ completed two modules, three (8.8\%) completed three modules and one $(2.9 \%)$ completed five modules. Twenty of the completers $(76.9 \%)$ provided follow-up data at the 3-month assessment, 16 (61.5\%) also did so at the 6-month assessment and 10 (38.5\%) even completed the

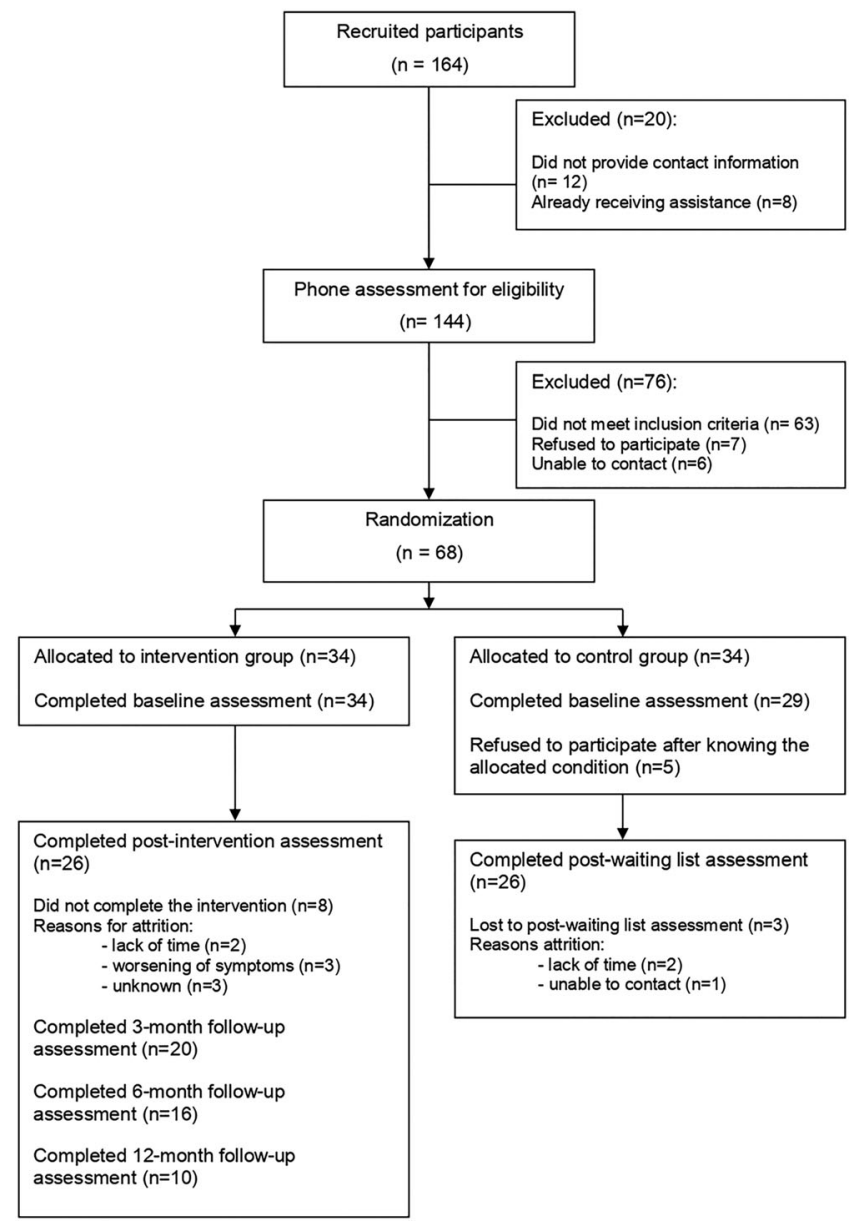

FIGURE 2 Participant flow diagram last 12-month follow-up assessment. Little's test confirmed the assumption that the data were missing completely at random $\left(\chi^{2}=56.46, \rho=1.000\right)$.

\section{2 | Baseline characteristics}

No baseline differences in demographic or clinical characteristics were found between groups. The overall sample consisted of 47 women (74.6\%) and 16 men (25.4\%) between 18 and 58 years of age $(M=32.81 ; S D=10.60)$, over half of whom were single $(54.0 \%$, $n=34)$. The majority of the participants were from Spain $(81.0 \%$, $n=51$ ), although people from other countries also participated. A high percentage of the sample were university students or people who had already completed their university education $(74.6 \%, n=47)$. Breakup, separation or divorce was the most frequently reported stressor $(30.2 \%, n=19)$, followed by conflicts with family members $(19.0 \%$, $n=12$ ) and problems in work or academic areas (19.0\%, $n=12)$. In almost half of the cases, the distress symptoms had been present for at least 6 months $(49.2 \%, n=31)$, but only eight participants were receiving medication at the time of assessment (12.7\%). Medication was monitored every week in order to detect any possible change in either the drug or the dosage. No changes in medication were reported.

Further details on demographic and clinical characteristics of the sample can be found in Tables 1 and 2 .

\section{3 | Treatment effectiveness at post-treatment}

Table 2 presents data on the intervention outcomes at post-treatment. A significant interaction effect of the moment of assessment (pretreatment and post-treatment) and the experimental condition (ICBT and WL) was found, indicating a different pattern of change between groups over time on the BDI $(\rho=0.001)$, ISL $(\rho=0.005)$, PTGI $(\rho=0.000)$, PANAS (positive affect: $\rho=0.001$; negative affect: $\rho=0.031)$ and MQLI $(\rho=0.024)$ scores. Compared with the control condition, the intervention group showed significantly more improvement on all the outcome measures (effect sizes from 0.54 to 1.21 ), except for the BAI ( $\rho=0.273 ; d=0.27$ ). Within-group comparisons showed significant improvements over time in participants in the ICBT group on all the measures considered, with preeffect-posteffect sizes ranging from 0.65 to 1.68 . However, participants in the control group only presented significant changes in their scores on the BDI and ISL. Within-group effect sizes in the control group ranged from 0.19 to 0.66 .

\subsection{Stability of treatment effects during the follow-up period}

Table 3 displays treatment effects in the intervention group at the follow-up assessments. The main effect of time was found to be 
TABLE 1 Baseline characteristics of participants

\begin{tabular}{|c|c|c|c|}
\hline Demographic and clinical characteristic & ICBT $(n=34)$ & WL $(n=29)$ & Between-group comparison \\
\hline Age mean $(S D)$ & $32.59(10.40)$ & $33.07(11.01)$ & $t(61)=0.18, \rho=0.859$ \\
\hline \multicolumn{4}{|l|}{ Gender, $n$ (\%) } \\
\hline Male & $10(29.4 \%)$ & $6(20.7 \%)$ & $\chi^{2}(1)=0.63, \rho=0.428$ \\
\hline \multicolumn{4}{|l|}{ Marital status, $n$ (\%) } \\
\hline Unmarried couple & $8(23.5 \%)$ & $4(13.8 \%)$ & \multirow{4}{*}{$\chi^{2}(4)=4.47, \rho=0.347$} \\
\hline Married & $6(17.6 \%)$ & $2(6.9 \%)$ & \\
\hline Divorced/separated & $3(8.8 \%)$ & $5(17.2 \%)$ & \\
\hline Widowed & 0 (0.0\%) & $1(3.4 \%)$ & \\
\hline \multicolumn{4}{|l|}{ Level of education, $n$ (\%) } \\
\hline \multicolumn{4}{|l|}{ Employment status, $n$ (\%) } \\
\hline Student & $14(41.2 \%)$ & $14(48.3 \%)$ & \multirow[t]{4}{*}{$\chi^{2}(3)=2.62, \rho=0.454$} \\
\hline Unemployed & $6(17.6 \%)$ & $3(10.3 \%)$ & \\
\hline Employed/self-employed & $12(35.3 \%)$ & $12(41.4 \%)$ & \\
\hline Work leave & $2(5.9 \%)$ & $0(0.0 \%)$ & \\
\hline \multicolumn{4}{|l|}{ Medication, $n$ (\%) } \\
\hline Yes & $6(17.6 \%)$ & 2 (6.9\%) & \multirow[t]{2}{*}{$\chi^{2}(1)=1.63, \rho=0.201$} \\
\hline No & $28(82.4 \%)$ & 27 (93.1\%) & \\
\hline$>6$ months & $13(38.2 \%)$ & $18(62.1 \%)$ & $\chi^{2}(3)=6.05, \rho=0.109$ \\
\hline Distress/interference severity, ${ }^{a}$ mean (SD) & $4.88(1.15)$ & $4.52(1.18)$ & $t(61)=1.24, \rho=0.220$ \\
\hline
\end{tabular}

Abbreviations: ICBT, Internet-delivered cognitive-behavioural therapy; SD, standard deviation; WL, waiting list.

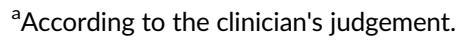

non-significant (all $\rho$ s $>0.053$ ), suggesting the overall stability of treatment gains achieved at post-treatment. Within-group effect sizes were even larger than at post-treatment, ranging from 0.92 to 2.12 at 3-month follow-up, from 0.85 to 2.05 at 6-month follow-up and from 0.81 to 2.04 at 12 -month follow-up.

\subsection{Clinically meaningful improvement: Reliable change}

Table 4 reports percentages of completer participants who showed clinically reliable change from baseline to postintervention. The percentage of participants who showed clinically reliable change was significantly higher in the intervention condition, compared with WL. These differences were significant for all the outcome measures. Percentages of participants who showed clinically significant change from baseline to different follow-up moments are also reported (see Table 3). Because of the small numbers and low power, no significance tests were conducted.

\section{4 | DISCUSSION}

The present study investigated the effectiveness of an ICBT intervention for AjD combined with brief weekly telephone support. The results showed that TAO was effective in reducing negative affect, as 
TABLE 2 Treatment outcomes at post-treatment

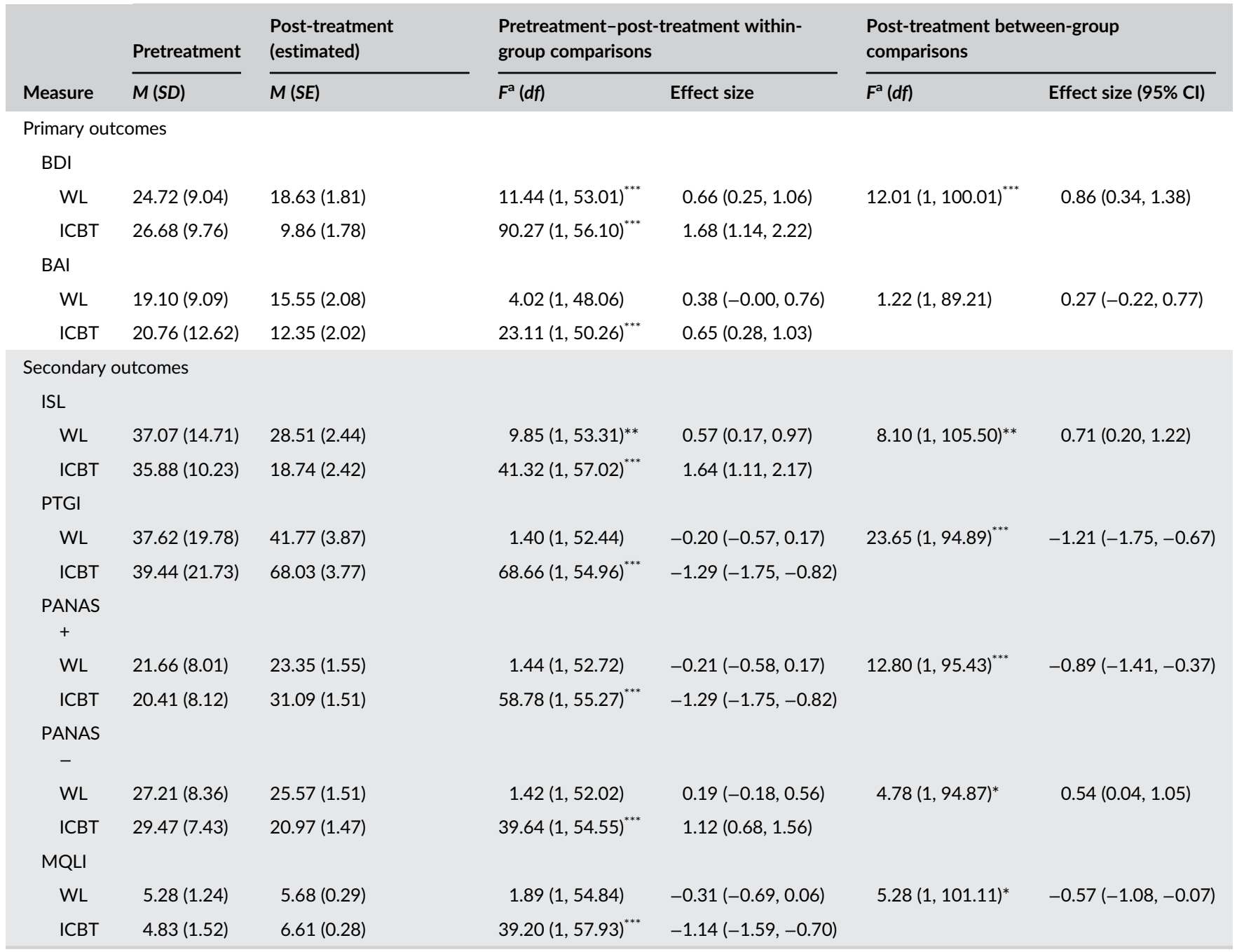

Abbreviations: BAI, Beck Anxiety Inventory; BDI, Beck Depression Inventory; Cl, confidence interval; df, degrees of freedom; ICBT, Internet-delivered cognitive-behavioural therapy; ISL, Inventory of Stress and Loss; M, mean; MQLI, Multidimensional Quality of Life Questionnaire; PANAS+, Positive and Negative Affect Scale-positive affect subscale; PANAS-, Positive and Negative Affect Scale-negative affect subscale; PTGI, Posttraumatic Growth Inventory; SD, standard deviation; SE, standard error; WL, waiting list.

${ }^{\mathrm{a}} \mathrm{F}$ values based on estimated means for interaction effects between time (pretreatment-post-treatment) and group (WL vs. ICBT).

${ }^{*} \rho \leq 0.050$.

$\rho \leq 0.010$.

$\rho \leq 0.001$

well as depressive, anxiety and AjD symptoms related to the stressor. The intervention also promoted post-traumatic growth, positive affect and quality of life.

Because AjD is considered to be a transitional, self-resolving condition (Bachem \& Casey, 2018), a reduction in clinical symptoms was also expected in control participants. As expected, the control group showed significant improvement but only on the outcome measures of depression (BDI) and AjD symptoms (ISL). Moreover, within-group effect sizes found in the control group were smaller than those found in the intervention group. Significant differences between the two groups were found. The intervention group presented significantly larger improvements on all the outcome measures, except on the BAI. The proportion of participants who showed reliable clinical change was also significantly higher in the
ICBT group. These results suggest that the impact of the stressful event diminished over time. However, the use of TAO not only promoted better adaptation to the stressful situation, reducing clinical symptoms triggered by the stressor, but it also facilitated positive changes such as post-traumatic growth and an increase in positive affect and quality of life.

The results of the trial also suggest the long-term stability of ICBT's effects. Nevertheless, despite being encouraging, these results must be viewed cautiously due to a high loss of data at follow-up and the lack of a control group at follow-up. Only $76.9 \%$ of the participants who completed the intervention provided 3-month follow-up data, and only $61.5 \%$ and $38.5 \%$ could be reached for the 6- and 12-month follow-up assessments, respectively. However, these results are in line with those found in the literature on the 


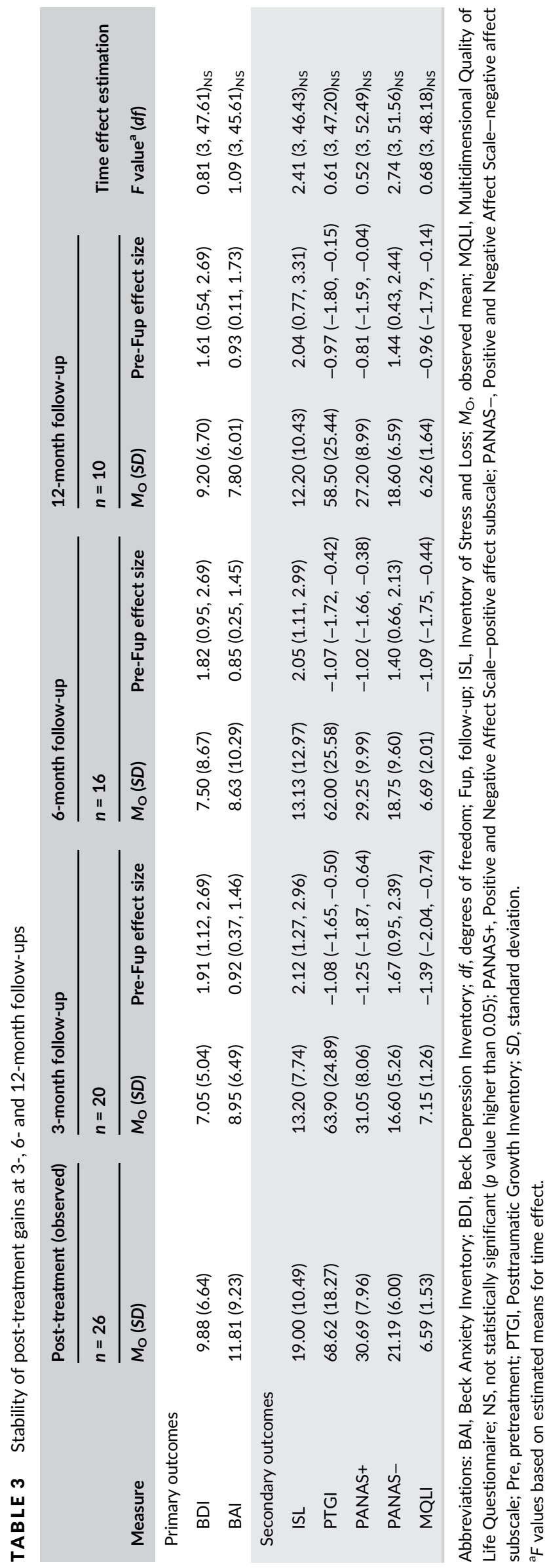

maintenance of ICBT treatment effects on AjD symptoms during the 3- and 6-month follow-up periods (Lindsäter et al., 2018; Moser et al., 2019)

The treatment adherence rate, that is, the proportion of participants who received the intended treatment, was $76.5 \%$, similar to what has been reported for other ICBT interventions combined with therapist support for adult depression and anxiety disorders (Andrews et al., 2018; Andrews, Cuijpers, Craske, McEvoy, \& Titov, 2010). Because both RCTs published to date on the effectiveness of completely unguided Internet interventions for AjD reported considerably higher attrition rates (Eimontas et al., 2017; Moser et al., 2019), it can be assumed that the weekly telephone support provided during the current trial played an important motivating role. Likewise, treatment adherence of $82 \%$ was found in individuals with AjD or exhaustion disorder who received ICBT combined with asynchronous online guidance from a therapist, which consisted of providing feedback about homework assignments and giving emotional and technical support (Lindsäter et al., 2018). Furthermore, in the present study, instead of giving a time interval to self-apply the program, modules were made available in a consecutive order, and so patients had to complete one module before accessing the next. Additionally, they also received weekly automated SMS reminders to log on to the treatment platform. These findings are consistent with the literature on the role of therapist support as a facilitator of adherence (Castro et al., 2018; Hilvert-Bruce, Rossouw, Wong, Sunderland, \& Andrews, 2012).

Although the dropout rate was low, the analyses suggested that the attrition appeared to be completely random. Moreover, in line with previous research, the dropouts occurred over the course of the treatment, rather than at the beginning (Van Ballegooijen et al., 2014). According to these authors, this gradual dropout pattern is not necessarily due to a lack of intervention acceptability, but rather, it might be explained by other factors, such as the feeling that the intervention is no longer needed. Nevertheless, further research is needed to draw firmer conclusions about factors related to adherence to ICBT interventions for AjD. Developing treatment programs that are attractive to patients will make it possible to reduce the current dropout rates.

\section{1 | Limitations}

The study has several limitations. First, most of the participants were recruited as volunteers who showed interest in an online intervention. It is unclear whether this willingness to receive an Internetdelivered intervention affected adherence and treatment outcomes. However, this interest is also encouraging and consistent with the existing literature. In a recent study, $25.0 \%$ of the participants reported a willingness to use self-help e-mental health services, and $33.8 \%$ indicated their intention to use therapist-assisted e-mental health programs (March et al., 2018). Another study found that the intention to use online mental health programs ranged between $40 \%$ and 71\% (Batterham \& Calear, 2017). Thus, Internet interventions 
TAB LE 4 Percentages of participants showing reliable change indexes

\begin{tabular}{|c|c|c|c|c|c|}
\hline & Post-treatment & Between-group comparisons & 3-month follow-up & 6-month follow-up & 12-month follow-up \\
\hline \multicolumn{6}{|c|}{ Primary outcomes } \\
\hline \multicolumn{6}{|l|}{$\mathrm{BDI}$} \\
\hline WL & $9(34.6 \%)$ & $\chi^{2}=9.43, \rho=0.002^{* *}$ & & & \\
\hline ICBT & 20 (76.9\%) & & 17 (85.0\%) & $15(93.8 \%)$ & $9(90.0 \%)$ \\
\hline WL & $2(7.6 \%)$ & $\chi^{2}=5.65, \rho=0.017^{*}$ & & & \\
\hline ICBT & $9(34.6 \%)$ & & $7(35.0 \%)$ & $5(31.3 \%)$ & $4(40.0 \%)$ \\
\hline \multicolumn{6}{|c|}{ Secondary outcomes } \\
\hline \multicolumn{6}{|l|}{ ISL } \\
\hline WL & $2(7.6 \%)$ & $\chi^{2}=5.65, \rho=0.017^{*}$ & & & \\
\hline ICBT & 9 (34.6\%) & & $4(20.0 \%)$ & $5(31.3 \%)$ & $2(20.0 \%)$ \\
\hline \multicolumn{6}{|c|}{ PANAS+ } \\
\hline WL & $2(7.7 \%)$ & $\chi^{2}=14.77, \rho=0.000^{* * *}$ & & & \\
\hline ICBT & $15(57.7 \%)$ & & $11(55.0 \%)$ & $7(43.8 \%)$ & $4(40.0 \%)$ \\
\hline \multicolumn{6}{|c|}{ PANAS- } \\
\hline WL & 1 (3.8\%) & $\chi^{2}=10.83, \rho=0.001^{* * *}$ & & & \\
\hline ICBT & $11(42.3 \%)$ & & $14(70.0 \%)$ & $10(62.5 \%)$ & $6(60.0 \%)$ \\
\hline \multicolumn{6}{|l|}{ MQLI } \\
\hline
\end{tabular}

Abbreviations: BAI, Beck Anxiety Inventory; BDI, Beck Depression Inventory; ICBT, Internet-delivered cognitive-behavioural therapy; ISL, Inventory of Stress and Loss; PANAS+, Positive and Negative Affect Scale-positive affect subscale; PANAS-, Positive and Negative Affect Scale-negative affect subscale; PTGI, Posttraumatic Growth Inventory; WL, waiting list.

${ }^{*} \rho \leq 0.050$.

${ }^{* *} \rho \leq 0.010$.

$\rho \leq 0.001$.

seem to represent an accepted treatment alternative for at least part of the population. Second, a large percentage of the sample $(74.6 \%)$ consisted of participants with a high educational level. Thus, the generalizability of the trial results may be compromised. The third limitation has to do with the measures of change in clinical symptoms. Only the ISL represented a specific measure of AjD symptoms. Although this instrument has already been validated and shows good psychometric properties (Quero, Mor, et al., 2019), inclusion of other disorder-specific measures, such as the Adjustment Disorders New Model questionnaire (Maercker et al., 2013a), would have provided more conclusive results. However, no validated instruments were available for the assessment of $A j D$ at the beginning of the RCT. Thus, none of the primary measures included the assessment of AjD specific symptoms. Instead, BDI and BAI were used because symptoms of depression and anxiety were identified as common manifestations of AjD (Maercker et al., 2013b). Although previous works support the utility of BDI to detect potential cases of AjD (Ruiz, Silva, \& Miranda, 2001), little is known about the utility of BAI. In the current study, BAI is the only instrument where no significant between-group differences were found. One possible explanation might be that BAI is not an appropriate instrument to assess AjD. As Leyfer, Ruberg, and Woodruff-Borden (2006) suggest, BAI might be a better measure of panic rather than of anxiety in general. Either way, further research is needed. It is also not clear whether the online administration of questionnaires affected their psychometric properties, because there are no studies on this subject. However, as we have mentioned before, good internal consistency indexes were found in the current sample for all outcome measures. The lack of an active treatment control group might be another study limitation. However, given the scarcity of evidence-based interventions for $A j D$, the comparison with a WL control group could be the first step in the validation of psychological treatments for this disorder. 
In addition, because $\mathrm{AjD}$ is considered a transient condition, it is especially useful to explore whether brief interventions like TAO can prevent the chronicity of this disorder and the development of more severe symptomatology. Finally, as mentioned previously, there was an important loss of data from postintervention to the follow-up assessment periods. Consequently, no firm conclusions can be drawn about the long-term durability of gains achieved with the ICBT delivered in the present study.

\section{2 | Conclusion}

The results of the present study support the efficacy of ICBT interventions such as TAO for the treatment of AjD. TAO was more effective than the mere passage of time in reducing the distress symptoms associated with this disorder, and it also conferred additional benefits in terms of post-traumatic growth, positive affect and quality of life. These results are consistent with those obtained with face-to-face implementation of the original treatment protocol (Quero, Molés, et al., 2019; Quero, Rachyla, et al., 2019). Thus, the current study makes an important contribution to the field of $\mathrm{AjD}$, indicating that the use of brief interventions with minimal therapist support can reduce the impact of one of the most common mental disorders. TAO might be an effective and accessible evidence-based treatment option for those who need psychological assistance but do not receive it due to long WLs.

\section{ACKNOWLEDGEMENTS}

The authors wish to thank the following institutions for the funding support that they provided to conduct this project: Ministerio de Economía y Competitividad (Spain; Plan Nacional I + D + I, PSI201341783-R); Plan 2018 de Promoción de la Investigación de la Universitat Jaume I (UJI-2018-57); Generalitat Valenciana pre-PhD grant program (VALi+d: ACIF/2015/181) and CIBERObn, an initiative of the Instituto de Salud Carlos III (ISCIII) (ISC III CB06 03/0052). The aforementioned institutions had no role in the design or execution of the study, analyses and interpretation of the data, or decision to submit results.

\section{CONFLICT OF INTERESTS}

The authors declare no conflicts of interest that could have influenced the work reported in this paper.

\section{AUTHOR CONTRIBUTION}

S. Q. formulated the research question together with C. B. I. R. analysed the data and drafted the manuscript, with important contributions from S. Q., P. C. and C. B. I. R., in collaboration with S. Q. and S. M., designed the study and participated in each of its phases. S. Q., I. R. and D. C. carried out the Internet-based adaptation of the treatment protocol. All authors participated in the review and revision of the manuscript and approved the final manuscript for publication.

\section{DATA AVAILABILITY STATEMENT}

The dataset used and/or analysed during the current study is available from the corresponding author on reasonable request.

\section{ORCID}

Pim Cuijpers (D) https://orcid.org/0000-0001-5497-2743

Cristina Botella (iD https://orcid.org/0000-0001-8783-6959

Diana Castilla (D) https://orcid.org/0000-0002-1631-1220

Soledad Quero (D) https://orcid.org/0000-0002-8973-1250

\section{REFERENCES}

American Psychiatric Association. (2013). Diagnostic and statistical manual of mental disorders (fifth ed.). Washington, DC: American Psychiatric Association. https://doi.org/10.1176/appi.books.9780890425596.744053

Andersson, G., \& Titov, N. (2014). Advantages and limitations of Internetbased interventions for common mental disorders. World Psychiatry, 13(1), 4-11. https://doi.org/10.1002/wps.20083

Andrews, G., Basu, A., Cuijpers, P., Craske, M. G., McEvoy, P., English, C. L., \& Newby, J. M. (2018). Computer therapy for the anxiety and depression disorders is effective, acceptable and practical health care: An updated meta-analysis. Journal of Anxiety Disorders, 55(August 2017), 70-78. https://doi.org/10.1016/j.janxdis.2018.01.001

Andrews, G., Cuijpers, P., Craske, M. G., McEvoy, P., \& Titov, N. (2010). Computer therapy for the anxiety and depressive disorders is effective, acceptable and practical health care: A meta-analysis. PLoS ONE, 5(10), e13196. https://doi.org/10.1371/journal.pone.0013196

Bachem, R., \& Casey, P. (2018). Adjustment disorder: A diagnosis whose time has come. Journal of Affective Disorders, 227, 243-253. https:// doi.org/10.1016/j.jad.2017.10.034

Bachem, R., \& Maercker, A. (2016). Self-help interventions for adjustment disorder problems: A randomized waiting-list controlled study in a sample of burglary victims. Cognitive Behaviour Therapy, 6073 (October), 1-17. https://doi.org/10.1080/16506073.2016.1191083

Baños, R. M., Guillen, V., Quero, S., García-Palacios, A., Alcaniz, M., \& Botella, C. (2011). A virtual reality system for the treatment of stressrelated disorders: A preliminary analysis of efficacy compared to a standard cognitive behavioral program. International Journal of Human Computer Studies, 69(9), 602-613. https://doi.org/10.1016/j.jijcs.2011.06.002

Batterham, P. J., \& Calear, A. L. (2017). Preferences for Internet-based mental health interventions in an adult online sample: Findings from an online community survey. JMIR Mental Health, 4(2), e26. https:// doi.org/10.2196/mental.7722

Beck, A. T., \& Steer, R. A. (1990). Manual for the Beck Anxiety Inventory. San Antonio, TX: Psychological Corporation.

Beck, A. T., Steer, R. A., \& Brown, G. K. (1996). Manual for the Beck Depression Inventory-II. San Antonio, TX: Psychological Corporation.

Berger, T., Boettcher, J., \& Caspar, F. (2014). Internet-based guided self-help for several anxiety disorders: A randomized controlled trial comparing a tailored with a standardized disorder-specific approach. Psychotherapy, 51(2), 207-219. https://doi.org/10.1037/a0032527

Berger, T., Hämmerli, K., Gubser, N., Andersson, G., \& Caspar, F. (2011). Internet-based treatment of depression: A randomized controlled trial comparing guided with unguided self-help. Cognitive Behaviour Therapy, 40(4), 251-266. https://doi.org/10.1080/16506073.2011.616531

Botella, C., Baños, R. M., \& Guillén, V. (2008). Creciendo en la adversidad. Una propuesta de tratamiento para los trastornos adaptativos. In C. Vázquez, \& G. Hervás (Eds.), Psicología Positiva Aplicada. Bilbao: DDB.

Carlbring, P., Andersson, G., Cuijpers, P., Riperd, H., \& HedmanLagerlöfh, E. (2018). Internet-based vs. face-to-face cognitive behavior therapy for psychiatric and somatic disorders: An updated systematic review and meta-analysis. Cognitive Behaviour Therapy, 47(1), 1-18. https://doi.org/10.1080/16506073.2017.1401115 
Casey, P., Jabbar, F., O'Leary, E., \& Doherty, A. M. (2015). Suicidal behaviours in adjustment disorder and depressive episode. Journal of Affective Disorders, 174, 441-446. https://doi.org/10.1016/j.jad.2014.12.003

Castro, A., López-del-Hoyo, Y., Peake, C., Mayoral, F., Botella, C., GarcíaCampayo, J., ... Gili, M. (2018). Adherence predictors in an Internetbased intervention program for depression. Cognitive Behaviour Therapy, 47(3), 246-261. https://doi.org/10.1080/16506073.2017.1366546

Chan, A.-W., Tetzlaff, J., Altman, D., Laupacis, A., Gøtzsche, P., \& Krleža-Jerić, K. (2013). SPIRIT 2013 statement: Defining standard protocol items for clinical trials. Research and Reporting Methods. Annals of Internal Medicine, 158(3), 200-207. https://doi.org/10.7326/00034819-158-3-201302050-00583

Chan, A. W., Tetzlaff, J. M., Gøtzsche, P. C., Altman, D. G., Mann, H., Berlin, J. A., ... Moher, D. (2013). SPIRIT 2013 explanation and elaboration: Guidance for protocols of clinical trials. BMJ (Clinical Research Ed.), 346, 1-42. https://doi.org/10.1136/bmj.e7586

Cohen, J. (1988). Statistical power analysis for the behavioral sciences (2nd ed.). Hillsdale, NJ: Lawrence Earlbaum Associates.

Cuijpers, P., Noma, H., Karyotaki, E., Cipriani, A., \& Furukawa, T. A. (2019). Effectiveness and acceptability of cognitive behavior therapy delivery formats in adults with depression: A network meta-analysis. JAMA Psychiatry, 76(7), 700-707. https://doi.org/10.1001/jamapsychiatry.2019.0268

Eimontas, J., Rimsaite, Z., Gegieckaite, G., Zelviene, P., \& Kazlauskas, E. (2017). Internet-based self-help intervention for ICD-11 adjustment disorder: Preliminary findings. Psychiatric Quarterly, 89, 1-10. https:// doi.org/10.1007/s11126-017-9547-2

Evans, S. C., Reed, G. M., Roberts, M. C., Esparza, P., Ritchie, P., \& Maj, M. (2013). Psychologists' perspectives on the diagnostic classification of mental disorders: Results from the WHO-IUPsyS Global Survey. International Journal of Psychology, 48(3), 177-193. https://doi.org/10. 1080/00207594.2013.804189

Eysenbach, G. (2011). CONSORT-EHEALTH: Improving and standardizing evaluation reports of Web-based and mobile health interventions. Journal of Medical Internet Research, 13(4), e126. https://doi.org/10. 2196/jmir.1923

Faul, F., Erdfelder, E., Buchner, A., \& Lang, A. G. (2007). G*Power 3: A flexible statistical power analysis program for the social, behavioral, and biomedical sciences. Behaviour Research Methods, 39(2), 175-191. https://doi.org/10.3758/BF03193146

Fegan, J., \& Doherty, A. M. (2019). Adjustment disorder and suicidal behaviours presenting in the general medical setting: $A$ systematic review. International Journal of Environmental Research and Public Health, 16(16). https://doi.org/10.3390/ijerph16162967

Fernández, A., Mendive, J. M., Salvador-Carulla, L., Rubio-Valera, M., Luciano, J. V., Pinto-Meza, A., ... Serrano-Blanco, A. (2012). Adjustment disorders in primary care: Prevalence, recognition and use of services. British Journal of Psychiatry, 201(2), 137-142. https://doi.org/ 10.1192/bjp.bp.111.096305

Griffiths, F., Lindenmeyer, A., Powell, J., Lowe, P., \& Thorogood, M. (2006). Why are health care interventions delivered over the internet? A systematic review of the published literature. Journal of Medical Internet Research, 8(2), 1-15. https://doi.org/10.2196/jmir.8.2.e10

Hilvert-Bruce, Z., Rossouw, P. J., Wong, N., Sunderland, M., \& Andrews, G. (2012). Adherence as a determinant of effectiveness of internet cognitive behavioural therapy for anxiety and depressive disorders. Behaviour Research and Therapy, 50(7-8), 463-468. https://doi.org/ 10.1016/j.brat.2012.04.001

Ivarsson, D., Blom, M., Hesser, H., Carlbring, P., Enderby, P., Nordberg, R., \& Andersson, G. (2014). Guided internet-delivered cognitive behavior therapy for post-traumatic stress disorder: A randomized controlled trial. Internet Interventions, 1(1), 33-40. https://doi.org/10.1016/j. invent.2014.03.002

Jacobson, N. S., \& Truax, P. (1991). Clinical significance: A statistical approach to defining meaningful change in psychotherapy research.
Journal of Consulting and Clinical Psychologv, 59(1), 12-19. https://doi. org/10.1037/0022-006X.59.1.12

Leyfer, O. T., Ruberg, J. L., \& Woodruff-Borden, J. (2006). Examination of the utility of the Beck Anxiety Inventory and its factors as a screener for anxiety disorders. Journal of Anxiety Disorders, 20(4), 444-458. https://doi.org/10.1016/j.janxdis.2005.05.004

Lindsäter, E., Axelsson, E., Salomonsson, S., Santoft, F., Ejeby, K., Ljótsson, B., ... Hedman-Lagerlöf, E. (2018). Internet-based cognitive behavioral therapy for chronic stress: A randomized controlled trial. Psychotherapy and Psychosomatics, 87(5), 296-305. https://doi.org/ 10.1159/000490742

Maercker, A., Bachem, R. C., Lorenz, L., Moser, C. T., \& Berger, T. (2015). Adjustment disorders are uniquely suited for eHealth interventions: Concept and case study. JMIR Mental Health, 2(2), e15. https://doi. org/10.2196/mental.4157

Maercker, A., Brewin, C. R., Bryant, R. A., Cloitre, M., Reed, G. M., Van Ommeren, M., ... Saxena, S. (2013a). Proposals for mental disorders specifically associated with stress in the International Classification of Diseases-11. The Lancet, 381(9878), 1683-1685. https://doi.org/10. 1016/S0140-6736(12)62191-6

Maercker, A., Brewin, C. R., Bryant, R. A., Cloitre, M., Van Ommeren, M., Jones, L. M., ... Reed, G. M. (2013b). Diagnosis and classification of disorders specifically associated with stress: Proposals for ICD-11. World Psychiatry, 12(3), 198-206. https://doi.org/10.1002/wps.20057

March, S., Day, J., Ritchie, G., Rowe, A., Gough, J., Hall, T., ... Ireland, M. (2018). Attitudes toward e-mental health services in a community sample of adults: Online survey. Journal of Medical Internet Research, 20(2), e59. https://doi.org/10.2196/jmir.9109

Mezzich, J. E., Ruiperez, M. A., Perez, C., Yoon, G., Liu, J., \& Mahmud, S. (2000). The Spanish version of the quality of life index:presentation and validation. The Journal of Nervous and MentalDisease, 188(5), 301-305. https://doi.org/10.1097/00005053-200005000-00008

Moher, D., Hopewell, S., Schulz, K. F., Montori, V., Gøtzsche, P. C., Devereaux, P. J., ... Altman, D. G. (2010). CONSORT 2010 explanation and elaboration: Updated guidelines for reporting parallel group randomised trials. BMJ (Clinical Research Ed.), 340, c869. http://www. pubmedcentral.nih.gov/articlerender.fcgi?artid $=2844943 \&$ tool $=$ pmcentrez\&rendertype=abstract, https://doi.org/10.1136/bmj.c869

Moser, C., Bachem, R., Berger, T., \& Maercker, A. (2019). ZIEL: Internetbased self-help for adjustment problems: Results of a randomized controlled trial. Journal of Clinical Medicine, 8(10), 1655. https://doi.org/ 10.3390/jcm8101655

Musiat, P., \& Tarrier, N. (2014). Collateral outcomes in e-mental health: A systematic review of the evidence for added benefits of computerized cognitive behavior therapy interventions for mental health. Psychological Medicine, 44(15), 3137-3150. https://doi.org/10.1017/ S0033291714000245

O'Donnell, M. L., Alkemade, N., Creamer, M., McFarlane, A. C., Silove, D., Bryant, R. A., ... Forbes, D. (2016). A longitudinal study of adjustment disorder after trauma exposure. American Journal of Psychiatry, 173 (12), 1231-1238. https://doi.org/10.1176/appi.ajp.2016.16010071

Piotrowski, C. (1999). The status of the Beck Anxiety Inventory in contemporary research. Psychological Reports, 85, 261-262. https://doi.org/ 10.2466/PRO.85.5.261-262

Prigerson, H. G., Maciejewsku, P. K., Reynolds, C. F., Bierhals, A. J., Newsom, J. T., Fasiczka, A., \& Miller, M. (1995). Inventory of complicated grief: A scale to measure maladaptive symptoms of loss. Psychiatry Research, 59, 65-79. https://doi.org/10.1016/0165-1781 (95)02757-2

Quero, S., Andreu-Mateu, S., Moragrega, I., Baños, R. M., Molés, M., Nebot, S., \& Botella, C. (2017). Un Programa Cognitivo-Conductual Que Utiliza La Realidad Virtual Para El Tratamiento De Los Trastornos Adaptativos: Una serie de casos. [A cognitive-behavioral therapy program utilizing virtual reality for the treatment of adjustment 
disorders: A case series]. Revista Argentina de Clínica Psicológica, 26(1), 5-18.

Quero, S., Molés, M., Campos, D., Andreu-Mateu, S., Baños, R. M., \& Botella, C. (2019). An adaptive virtual reality system for the treatment of adjustment disorder and complicated grief: 1-year follow-up efficacy data. Clinical Psychology \& Psychotherapy, 26, 204-217. https:// doi.org/10.1002/cpp.2342

Quero, S., Mor, S., Molés, M., Rachyla, I., Baños, R. M., \& Botella, C. (2019). Exploring the assessment of adjustment disorders: Differences between a general and a clinical sample. Psicothema, 31(1), 17-23. https://doi.org/10.7334/psicothema2018.159

Quero, S., Rachyla, I., Molés, M., Mor, S., Tur, C., Cuijpers, P., ... Botella, C. (2019). Can between-session homework be delivered digitally? A pilot randomized clinical trial of CBT for adjustment disorders. International Journal of Environmental Research and Public Health, 16(20). https:// doi.org/10.3390/ijerph16203842

Rachyla, I., Pérez-Ara, M., Molés, M., Campos, D., Mira, A., Botella, C., \& Quero, S. (2018). An internet-based intervention for adjustment disorder (TAO): Study protocol for a randomized controlled trial. BMC Psychiatry, 18(1), 161. https://doi.org/10.1186/s12888-018-1751-6

Richter, P., Werner, J., Heerlein, A., Kraus, A., \& Sauer, H. (1998). On the validity of the Beck Depression Inventory. A review. Psychopathology, 31(3), 160-168. https://doi.org/10.1159/000066239

Ruiz, A., Silva, H., \& Miranda, E. (2001). Clinical and psychometric diagnosis of depression in general medical patients. Revista Médica de Chile, 129 (6), 627-633. https://doi.org/10.4067/S0034-98872001000600005

Salim, A., Mackinnon, A., Christensen, H., \& Griffiths, K. (2008). Comparison of data analysis strategies for intent-to-treat analysis in pre-test-post- test designs with substantial dropout rates. Psychiatry Research, 160(3), 335-345. https://doi.org/10.1016/j.psychres.2007.08.005

Tadeschi, R. G., \& Calhoun, L. G. (1996). The Posttraumatic Growth Inventory: Measuring the positive legacy of trauma. Journal of Traumatic Stress, 9(3), 455-471. https://doi.org/10.1002/jts.2490090305

Van Ballegooijen, W., Cuijpers, P., Van Straten, A., Karyotaki, E., Andersson, G., Smit, J. H., \& Riper, H. (2014). Adherence to internetbased and face-to-face cognitive behavioural therapy for depression: A meta-analysis. PLoS ONE, 9(7), e100674. https://doi.org/10.1371/ journal.pone.0100674

Watson, D., Clark, L. A., \& Tellegen, A. (1988). Development and validation of brief measures of positive and negative affect: The PANAS scales. Journal of Personality and Social Psychology, 54(6), 1063-1070. https:// doi.org/10.1037/0022-3514.54.6.1063

World Health Organization. (2018). International Classification of Diseases for Mortality and Morbidity Statistics, Eleventh Revision. Retrieved from https://icd.who.int

How to cite this article: Rachyla I, Mor S, Cuijpers P, Botella C, Castilla D, Quero S. A guided Internet-delivered intervention for adjustment disorders: A randomized controlled trial. Clin Psychol Psychother. 2020;1-12. https://doi.org/10.1002/ cpp. 2518 